\title{
Thermocycling on the Web: A user's guide
}

\section{Richard Peters and Robert Sikorski}

Imagine walking into the world's largest library, stepping up to the card catalog, and finding that it consisted of scraps of paper containing the first paragraph of every document in the library. To find the information you are after, you have to guess what keyword will be contained in the first paragraph of the document you suppose the library is holding. Since the documents are constantly being updated, and their locations also move, the only way to find what you are after is to go to the general location of all the documents that have a specific keyword you are

Richard Peters and Robert Sikorski are Howard Hughes Medical Institute fellows at Harvard Medical School and the National Cancer Institute, respectively (rpeters@vp3.med.harvard.edu and rss@nchgr.nih.gov). looking for, and then open them one by one.

If this sounds complicated, then you have an inkling of what researchers are up against when they try to find specific information on the World Wide Web. Since there is no road map to the type or quality of information available on the Web, searching is a hit or miss proposition. If you are lucky, you can find exactly what you are after in a few keystrokes, but if the topic does not lend itself to highly specific keywords that a search engine can find-in the first few paragraphs of a Web page-then you may be in for a highly frustrating experience.

A quick search on the term "PCR" is a case in point. Searching with a typical search engine will generate over 600 different documents. Which ones are accurate, up-to-date, and contain the information you need?

As MDs doing research, we were doubly frustrated by this problem: We wanted the latest medical information for treating our patients (Science 270:16-17, 1995), as well as the most up-to-the-minute information on our specific areas of research. Although the Web seemed to be the obvious place to get this type of data, we did not have the hours it would require to search through each site to find these gems. In an effort to make biomedical information accessible quickly, we developed a search software that we call MIRIAD, which enables rapid filtering for all documents available on the Web on a specific topic [Ed.: contact authors for additional information]. The table below gives one search result. Of 600 documents found, these 10 sites were filtered for high-quality information on PCR. These sites should help you get your bearings for ferreting out the specific PCR information you are after.

\section{PCR PRODUCT AND SERVICES SITES}

\section{Page Name \\ PCR reagents/instruments LAPCR \\ p8-10.pdf \\ Long PCR reagents \\ URL \\ http://www.perkin-elmer.com/abd/pcr/ \\ http://www.panvera.com/webpages/laguide.html \\ http://biochem.boehringer.com/prod_inf/manuals/pcr_man/p8-10.pdf http://twod.med.harvard.edu/labgc/estep//ongPCR_protocol.html}

Suppliers of PCR products PCR in situ

Primer selection

PRIMER

Molecular biology techniques Protocols on the WuW

http://www.the-scientist.library.upenn.edu/yr1996/feb/pcr_960205.html http://lenti.med.umn.edu/insitu_pcr/chap-shoot2.fm.html http://alces.med.umn.edu/webprimers.html

http://www.csc.fi/molbio/progs/primer/manual.html

http://research.nwfsc.noaa.gov/protocols/methods/methods.html http://www.ifrn.bbsrc.ac.uk/gm/lab/docs/protocols.html

\section{Comments}

Has a nice frequently asked questions section A good reference for long-range PCR A general manual for standard PCR techniques A protocol for long PCR from the laboratory of

George Church at Harvard Medical School A good review of products available for PCR

A good description of in situ PCR

A program to aid in selecting PCR primers from the University of Minnesota

A program to help in selecting PCR Primers from the MIT Genome Center

A forum for exchanging PCR techniques A hotlist of molecular biology techniques
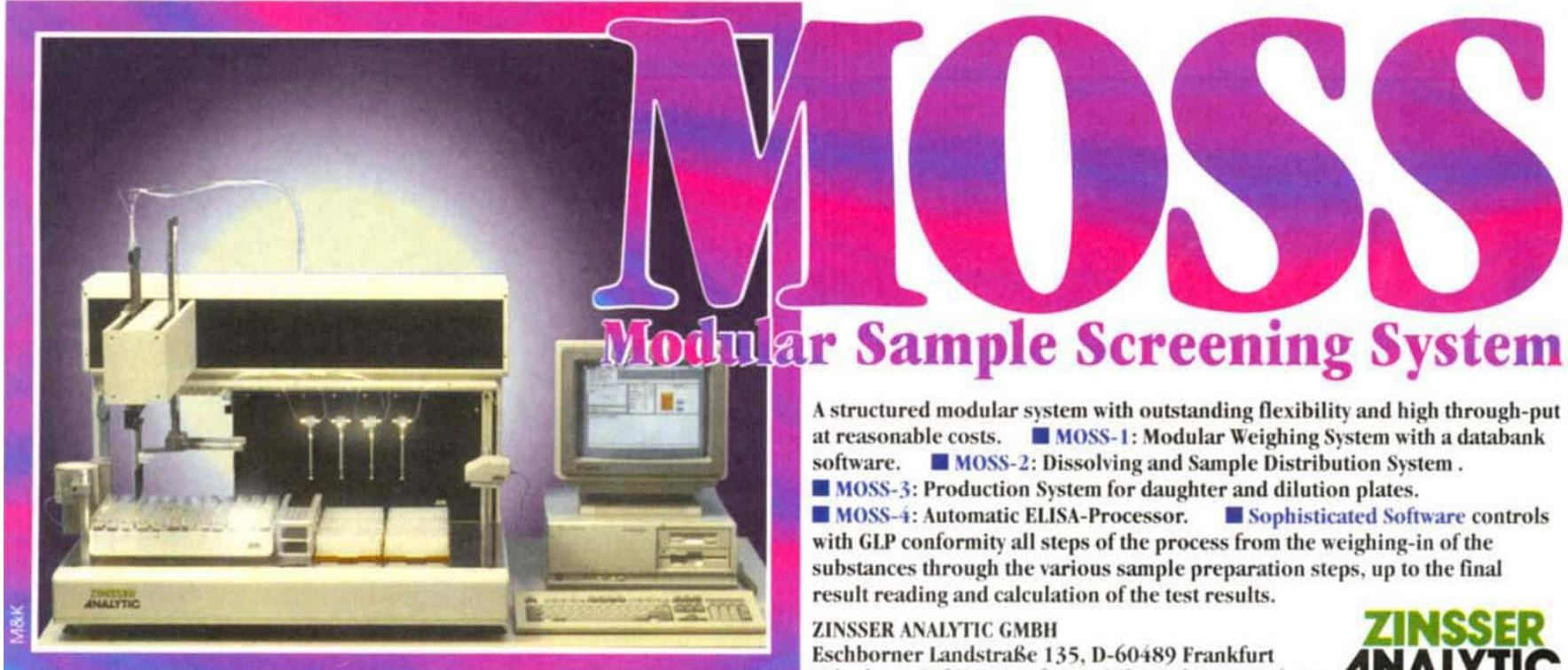

A structured modular system with outstanding flexibility and high through-put at reasonable costs. $\quad$ MOSS-1: Modular Weighing System with a databank software. MOSS-2: Dissolving and Sample Distribution System . noss-3: Production System for daughter and dilution plates.

MOSS-4: Automatic EL.ISA-Processor. E Sophisticated Software controls with GL.P conformity all steps of the process from the weighing-in of the substances through the various sample preparation steps, up to the final result reading and calculation of the test results.

ZINSSER ANALYTIC GMBH

Eschborner Landstraße 135, D-60489 Frankfurt

Telephone (069)789106-0, Telefax (069)789106-80 